\title{
Reactor de flujo continuo para la remoción del plomo por electrocoagulación en las aguas del río Coata, Puno-Perú
}

Continuous flow reactor for the removal of lead by electrocoagulation un the waters of the Coata river, Puno-Peru

Recibido: agosto 03 de 2021 | Revisado: abril 13 de 2021 | Aceptado: mayo 10 de 2021

\author{
Russel Allidren Lozada Vilca ${ }^{\mathrm{I}}$ \\ George Argota Pérez ${ }^{\mathrm{I}}$ \\ Edson Ramos Ninaja ${ }^{\mathrm{I}}$ \\ Rony Milton Choqueneira Ccama ${ }^{\mathrm{I}}$
}

\begin{abstract}
Resumen
El objetivo fue diseñar un reactor de flujo continuo para la remoción experimental del plomo por electrocoagulación en las aguas del río Coata de Puno, Perú. El diseño del reactor consistió en dos celdas de flujo continuo que se conectaron por un canal de tuberías. Las dimensiones de las celdas fueron las siguientes: $17 \times 20 \times 30 \mathrm{~cm}$. Los electrodos con medición de 23 $\mathrm{x} 11 \mathrm{~cm}$. y con un adicional de $1 \mathrm{~cm}^{2}$. Asimismo, la fuente de voltaje fue de $50 \mathrm{Vdc}$ con una densidad de corriente de $0.8 \mathrm{~A}$. El ángulo de salida para el fluido fue de $45^{\circ}$ donde existió un caudal de $0.008 \mathrm{~L} / \mathrm{s}$ con 18.36 minutos de tiempo de retención en la primera celda y $34.68 \mathrm{~min}$ en la segunda, respectivamente. La concentración inicial del plomo en la muestra de aguas sin tratamiento fue de $0.081 \mathrm{mg} / \mathrm{L}^{-1}$. Para la primera celda, la concentración que se halló fue de 0.038 $\mathrm{mg} / \mathrm{L}^{-1}$, mientras que, $0,011 \mathrm{mg} / \mathrm{L}^{-1}$ correspondió a la segunda celda. Los resultados indican un porcentaje de remoción del $53 \%$ y $86 \%$ para cada celda. Se concluye que, el diseńo del rector de flujo continuo removió de forma satisfactoria y en condiciones experimentales las concentraciones de plomo en las aguas del río Coata de Puno, Perú.
\end{abstract}

Palabras clave: agua, contaminación, electrocoagulación, plomo, río Coata

\begin{abstract}
The aim was to design a continuous flow reactor for the experimental removal of lead by electrocoagulation in the waters of the Coata River in Puno, Peru. The reactor design consisted of two flow-through cells that were connected by a pipe channel. The dimensions of the cells were as follows: $17 \times 20 \times 30 \mathrm{~cm}$. The electrodes measured $23 \times 11 \mathrm{~cm}$. and with an additional $1 \mathrm{~cm}^{2}$. Likewise, the voltage source was $50 \mathrm{Vdc}$ with a current density of $0.8 \mathrm{~A}$. The exit angle for the fluid
\end{abstract}

1 Universidad Peruana Unipon (UPeU). Juliaca, Puno-Perú. lessurlv2@yahoo.es

2 Centro de Investigaciones Avanzadas y Formación Superior en Educación, Salud y Medio Ambiente "AMTAWI". Perú george.argota@gmail.com

(C) Los autores. Este artículo es publicado por la Revista Campus de la Facultad de Ingeniería y Arquitectura de la Universidad de San Martín de Porres. Este artículo se distribuye en los términos de la Licencia Creative Commons Atribución No-comercial - Compartir-Igual 4.0 Internacional (https://creativecommons.org/licenses/ CC-BY), que permite el uso no comercial, distribución y reproducción en cualquier medio siempre que la obra original sea debidamente citada. Para uso comercial contactar a: revistacampus@usmp.pe. 
was $45^{\circ}$ where there was a flow of $0.008 \mathrm{~L} / \mathrm{s}$ with 18.36 minutes of retention time in the first cell. and $34.68 \mathrm{~min}$ in the second, respectively. The initial concentration of lead in the untreated water sample was $0.081 \mathrm{mg} / \mathrm{L}^{-1}$. For the first cell, the concentration found was 0.038 $\mathrm{mg} / \mathrm{L}^{-1}$, while $0.011 \mathrm{mg} / \mathrm{L}^{-1}$ corresponded to the second cell. The results indicate a removal percentage of $53 \%$ and $86 \%$ for each cell. It is concluded that the design of the continuous flow rector satisfactorily removed lead concentrations in the waters of the Coata River in Puno, Peru under experimental conditions.

Key words: Coata River, electrocoagulation, lead, pollution, water

\section{Introducción}

La exposición no regulada de elementos químicos en los cuerpos de agua genera problemas ambientales a todos los organismos, así como al propio ecosistema (Deng et al., 2017; Chai et al., 2017), por cuanto se necesita monitorear la calidad del agua para evaluar si es adecuada según el valor de uso que se desee incluyendo su servicio potable (Allaire et al., 2018; Paul et al., 2019). Entre los elementos químicos se encuentra el plomo y se conoce los múltiples daños que ocasiona a la salud humana, pero lo significativo es el deterioro al sistema nervioso central y que repercute sobre las funciones cognitivas e intelectuales, fundamentalmente, en fetos y niños (as) (Jusko et al., 2008). Las tuberías de plomo que se conectan al sistema o red de plomería doméstica para el servicio público son una de las principales fuentes de exposición (Sandvig et al., 2008; Del Toral et al., 2013). Por ejemplo, en los EE.UU. se estima que existen 6.1 millones de tuberías de plomo (Cornwell et al., 2016) y entre 6.5 a 10 millones de hogares prescinden de líneas de servicios que estarían fabricadas de plomo (EPA,
2016). Si las fuentes de suministro del agua y las operaciones para su tratamiento no se planifican de manera eficiente, siempre ocurrirá la liberación excesiva de plomo (Pieper et al., 2018; Roy \& Edwards, 2019).

En los últimos años, existe gran interés por parte de la comunidad científica en la búsqueda de métodos eficientes para la eliminación de metales pesados de las aguas residuales y otras fuentes de uso de la población humana. Una de las técnicas es la electrocoagulación donde se utiliza un reactor autocatalítico para mejorar la cinética de adsorción y con ello, eliminarse el metal de interés (AlJaberi, 2020). La eliminación de los metales se produce por la presencia de ánodos convencionales tales como el aluminio, hierro y el cobre. Sin embargo, estos sistemas tienen la desventaja de ser costosos debido a su requerimiento de energía eléctrica y los propios materiales de electrodos los cuales influyen de modo directo en eficiencia de la electrocoagulación (Hussin etal., 2017). El objetivo fue diseñar un reactor de flujo continuo para la remoción experimental del plomo por electrocoagulación en las aguas del río Coata de Puno, Perú. 


\section{Métodos}

El estudio se realizó en el río Coata de la ciudad de Juliaca, provincia de San Román, departamento de Puno, Perú. Se seleccionó una zona de exposición (E: 385447, N: 828538719 L UTM) para el muestreo probabilístico aleatorio de las aguas (ISO, 1980, 1991, 1994).

Se determinó en el Laboratorio Acreditado "RHLAB S.A.C." la concentración total $\left(\mathrm{mg} / \mathrm{L}^{-1}\right)$ de plomo en la que su comparación normativa fue según el Decreto Supremo N004-2017MINAM, Categoría 1. Para el tratamiento de las muestras de aguas se diseñó un reactor de flujo continuo (Figura 1) con las características siguientes:

a. Dos celdas de flujo continuo que se conectaron por un canal de tuberías.

b. Las dimensiones de las celdas fueron: $17 \times 20 \times 30 \mathrm{~cm}$.

c. Electrodos con medición de $23 \times 11$ $\mathrm{cm}$. y con un adicional de $1 \mathrm{~cm}^{2}$.

d. Fuente de voltaje fue de $50 \mathrm{Vdc}$ con una densidad de corriente de $0.8 \mathrm{~A}$.

e. Ángulo de salida para el fluido fue de $45^{\circ}$

f. Caudal de $0.008 \mathrm{~L} / \mathrm{s}$ con 18.36 minutos de tiempo de retención en la primera celda y $34.68 \mathrm{~min}$ en la segunda.

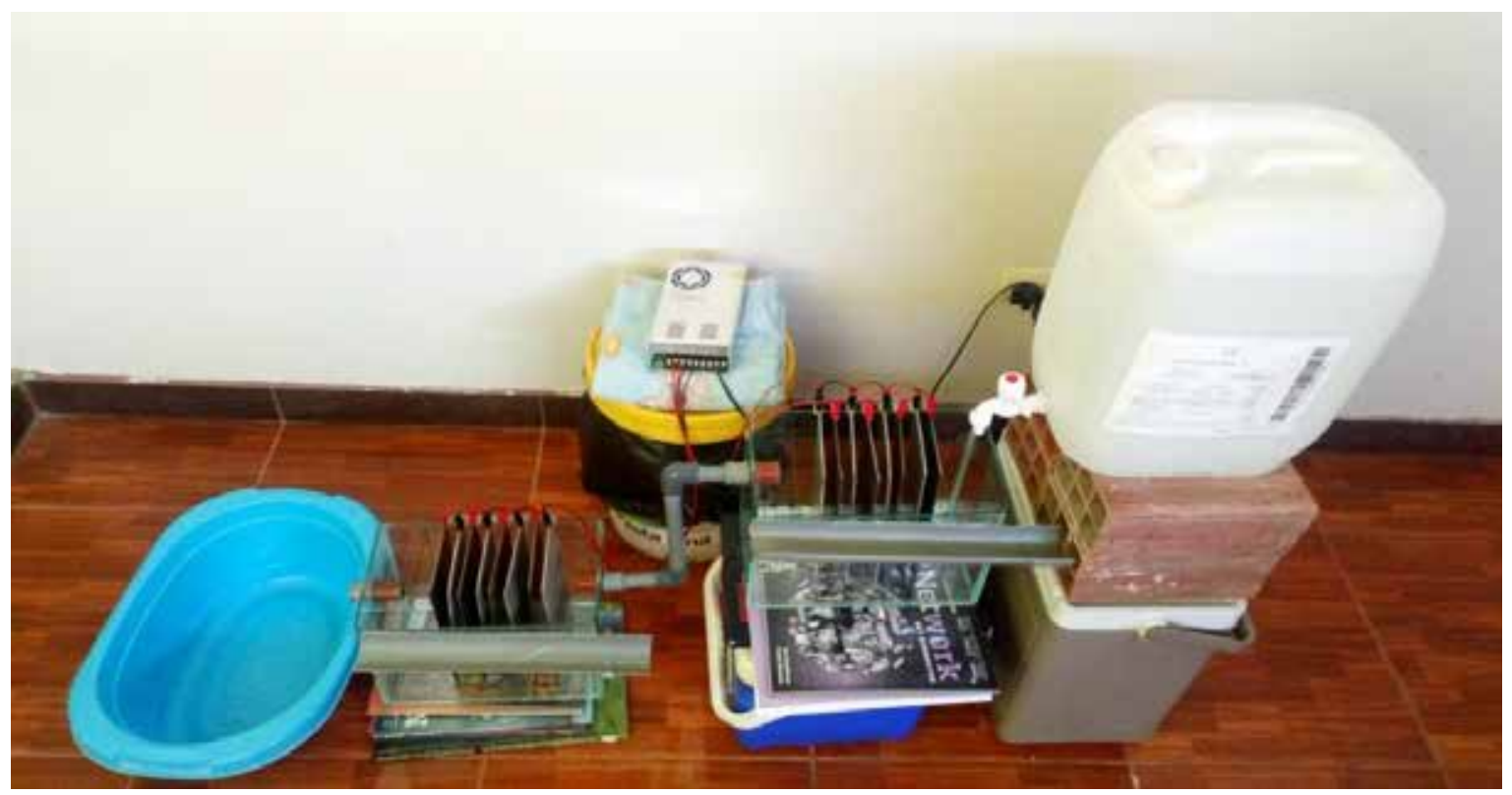

Figura 1. Reactor de tipo continuo / tratamiento de plomo / matriz agua

Seanalizóel porcentaje(\%)deremoción del plomo por electrocoagulación entre las celdas 1 y 2 para estimar la eficiencia del reactor de flujo continuo según la concentración que establece la norma ambiental que se utilizó.

\section{Resultados y Discusión}

La Tabla 1 muestra la concentración total promedio $\left(\mathrm{mg} / \mathrm{L}^{-1}\right)$ de las muestras de aguas procedente del río Coata y el valor que establece Decreto Supremo (DS) Nº04-2017-MINAM. 
Tabla 1

Concentración total de plomo $\left(m g / L^{-1}\right) /$ rio Coata

\begin{tabular}{cccc}
\hline Elemento & Réplicas & Concentración & DS No004-2017-MINAM \\
\hline \multirow{2b}{*}{} & 1 & 0.079 & \\
& 2 & 0.081 & 0.5 \\
\hline
\end{tabular}

La Tabla 2 muestra el porcentaje de remoción por electrocoagulación para cada celda de flujo continuo donde sus valores se encontraron por debajo del límite máximo permisible según la norma ambiental que se utilizó.

Tabla 2

Porcentaje de remoción (\%) / reactor de flujo continuo

\begin{tabular}{ccccc}
\hline Elemento & $\begin{array}{c}\text { Celda de flujo } \\
\text { continuo }\end{array}$ & $\begin{array}{c}\text { Concentración } \\
\text { total }\end{array}$ & Porcentaje & $\begin{array}{c}\text { DS N } \text { N004-2017- }^{\circ} \\
\text { MINAM }\end{array}$ \\
\hline \multirow{2}{*}{$\mathrm{Pb}$} & 1 & $0.038 \pm 0,003$ & 53.09 & 0.5 \\
& 2 & $0.011 \pm 0,002$ & 86.42 & \\
\hline
\end{tabular}

Los metales pesados generan efectos perjudiciales y en las últimas décadas se tiene mayor conciencia para la preservación de los ecosistemas acuáticos (Aji et al., 2012). Ante la contaminación del agua, la comunidad científica enfrenta como nuevos desafíos la búsqueda de métodos confiables y rentables para su tratamiento (Hakizimana et al., 2017). Uno de estos métodos es la electrocoagulación ya que al no requerirse de elementos químicos, tampoco se producen efectos secundarios (Moussa et al., 2017), pero debe mencionar que la electrocoagulación asistida por otros procesos de tratamientos avanzados contribuye a mejorar la calidad de los efluentes (AlQodah et al., 2018; AlQodah, Al-Qudah \& Omar, 2019).

En algunos estudios como el que practicó El-Ashtoukhy et al., (2020) se aplicó gas propio, un ánodo de lámina de $\mathrm{Al}^{+}$cilíndrico y un cátodo de pantalla de igual material y colocado a una pequeña distancia del ánodo. Se halló porcentajes de remoción entre el 34,56 y $100 \%$ para otros metales como el $\mathrm{Cu}_{2}{ }^{+}$,
$\mathrm{Ni}_{2}^{+}$. Asimismo, diversos estudios en la literatura científica pueden contrastarse, pero las condiciones experimentales que se ensayaron en este estudio indicaron que la electrocoagulación que realizó mediante el diseño del reactor de flujo continuo proporcionó un tratamiento adecuado cumpliéndose con el requerimiento del nivel permisible por la norma ambiental que se utilizó.

En el artículo de revisión que realizó Mouzza et al., (2017) y que se refirió a la electrocoagulación para el tratamiento de las aguas residuales: potenciales y desafíos se indicó que, su alta eficiencia contra contaminantes inorgánicos $\mathrm{u}$ orgánicos incluidos los persistentes $y$ que se encuentran desde las aguas subterráneas hasta los efluentes de refinerías. Asimismo, se señala que la mayor parte de las evaluaciones se centran en contaminantes específicos sin destacar tipos de diseños, modelación a procesos y aplicaciones industriales.

Existió como principal limitación del estudio, la determinación de otros 
metales pesados, además, de evaluarse diferentes procesos electroquímicos que refieran al voltaje, densidad de la corriente, distancia, tipo de conexión, tamaño y área activa de los electrodos para luego indicar algunas características fisicoquímicas del electrolito y que refiera al $\mathrm{pH}$, conductividad eléctrica y la temperatura que son parámetros físico- químicos influyentes en la especiación química de los metales pesados.

Se concluye que el diseño del reactor de flujo continuo removió en condiciones experimentales y mediante el proceso de electrocoagulación el plomo de las aguas del río Coata de Puno, Perú.

\section{Referencias}

Al Aji, B., Yavuz, Y. \& Koparal, A.S. (2012). Electrocoagulation of heavy metals containing model wastewater using monopolar iron electrodes. Sep. Purif. Technol; 86, 248-254. https://doi. org/10.1016/j.seppur.2011.11.011

AlJaberi, F.Y. (2020). Corrigendum to "Studies of autocatalytic electrocoagulation reactor for lead removal from simulated wastewater" J. Environ. Chem. Eng; 8(3), 6069-6078. https://doi. org/10.1016/j.jece.2019.103511

Allaire, M., Wu, H. \& Lall, U. (2018). National trends in drinking water quality violations. Proc. Natl. Acad. Sci; 115(9), 2078-2083. https://doi. org/10.1073/pnas.1719805115

Al-Qodah, Z., Al-Qudah, Y. \& Omar, W. (2019). On the performance of electrocoagulationassisted biological treatment processes: a review on the state of the art. Environ. Sci. Poll. Res; 26, $28689-$ 28713. https://doi.org/10.1007/ s11356-019-06053-6

AlQodah, Z., Al-Shannag, M., BaniMelhem, K., Assirey, E.,
Yahya, M.A. \& AlShawabkeh, A. (2018). Free radicalassisted electrocoagulation processes for wastewater treatment. Environ. Chem. Lett; 16, 695-714. https:// doi.org/10.1007/s10311-0180711-1

Chai, L., Chen, A., Luo, P., Zhao, H. \& Wang, H. (2017). Histopathological changes and lipid metabolism in the liver of Bufo gargarizans tadpoles exposed to Triclosan. Chemosphere; 182, 255266. https://doi.org/10.1016/j. chemosphere.2017.05.040

Cornwell, D.A., Brown, R.A. \& Via, S.H. (2016). National survey of lead service line occurrence. J. Am. Water Work. Assoc; 108(4), 182191. https://doi.org/10.5942/ jawwa.2016.108.0086

Decreto Supremo No004-2017MINAM. Aprueban Estándares de Calidad Ambiental (ECA) para Agua y establecen Disposiciones Complementarias. Consultado 2-febrero-2021. https://www. minam.gob.pe/disposiciones/ decreto-supremo-n-004-2017minam/ 
Del Toral, M.A., Porter, A. \& Schock, M.R. (2013). Detection and evaluation of elevated lead release from service lines: a field study. Environ. Sci. Technol; 47(16), 93009307. https://doi.org/10.1021/ es4003636

Deng, H., Chai, L., Luo, P., Zhou, M., Nover, D. \& ZhaO, X. (2OI7). TOXIC EFFects of $\mathrm{NH}_{4}+-\mathrm{N}$ on embryonic development of Bufo gargarizans and Rana chensinensis. CHEMOSPHERE; 182, 617-623. HTTPS://DOI.ORG/IO.IOI 6/J. CHEMOSPHERE.2OI7.02.1 56

El-Ashtoukhy, E.-S. Z., Amin, N. K., Fouad, Y. O. \& Hamad, H. A. (2020). Intensification of a new electrocoagulation system characterized by minimum energy consumption and maximum removal efficiency of heavy metals from simulated wastewater. Chemical Engineering and Processing - Process Intensification; 154, 2-12. https://doi:10.1016/j. cep. 2020.108026

EPA. (2016). Lead and Copper Rule Revisions White Paper. United States Environ. Prot. Agency (October), 1-18.

Hakizimana, J.N., Gourich, B., Chafi, M., Stiriba, Y., Vial, C., Drogui, P. \& Naja, J. (2017). Electrocoagulation process in water treatment: a review of electrocoagulation modeling approaches. Desalination; 404, 1-21. https://doi.org/10.1016/j. desal.2016.10.011
Hussin, F., Abnisa, F., Issabayeva, G. \& Aroua, M.K. (2017). Removal of lead by solar-photovoltaic electrocoagulation using novel perforated zinc electrode. Journal of Cleaner Production; 147, 206$216 . \quad$ https://doi:10.1016/j. jclepro.2017.01.096

Jusko, T.A., Charles Jr., R.H., Lanphear, B.P., Cory-Slechta, D.A., Parsons, P.J. \& Canfield, R.L. (2008). Blood lead concentrations < $10 \mathrm{micrograms} / \mathrm{dL}$ and child intelligence at 6 Years of age. Environ. Health Perspect; 116(2), 243-248. https://dx.doi. org/10.1289\%2Fehp.10424

Moussa, D.T., El-Naas, M.H., Nasser, M. \& Al-Marri, M.J. (2017). A comprehensive review of electrocoagulation for water treatment: potentials and challenges, J. Environ. Manage; 186, 24-41. https://doi.org/10.1016/j. jenvman.2016.10.032

Normalization Standart International: ISO 5667-1. (1980). Water quality. Sampling. Part 1: Guidance on the design of sampling programmes.

Normalization Standart International: ISO 5667-2. (1991). Water quality. Sampling. Part 2: Guidance on sampling techniques.

Normalization Standart International: ISO 5667-3. (1994). Water quality. Sampling. Part 3: Guidance on the preservation and handling of samples. 
Paul, S., Mandal, A., Bhattacharjee, P., Chakraborty, S., Paul, R. \& Kumar Mukhopadhyay, B. (2019). Evaluation of water quality and toxicity after exposure of lead nitrate in fresh water fish, major source of water pollution. The Egyptian Journal of Aquatic Research; 45(4), 345-351. https://doi. org/10.1016/j.ejar.2019.09.001

Pieper, K.J., Martin, R., Tang, M., Walters, L., Parks, J., Roy, S., Devine, C. \& Edwards, M.A. (2018). Evaluating water lead levels during the flint water crisis. Environ. Sci. Technol; 52(15), 8124-8132. https://doi. org/10.1021/acs.est.8b00791
Roy, S. \& Edwards, M.A. (2019). Preventing another lead $(\mathrm{Pb})$ in drinking water crisis: lessons from the Washington D.C. and Flint MI contamination events. Curr. Opin. Environ. Sci. Heal; (7), 3444. https://doi.org/10.1016/j. coesh.2018.10.002

Sandvig, A., Kwan, P., Kirmeyer, G., Maynard, B., Mast, D., Trussell, R.R., Trussell, S., Cantor, A. \& Prescott, A. (2008). Contribution of Service Line and Plumbing Fixtures to Lead and Copper Rule Compliance Issues, vol. 523. Awwa Res. Found. - US Environ. Prot. Agency. 
